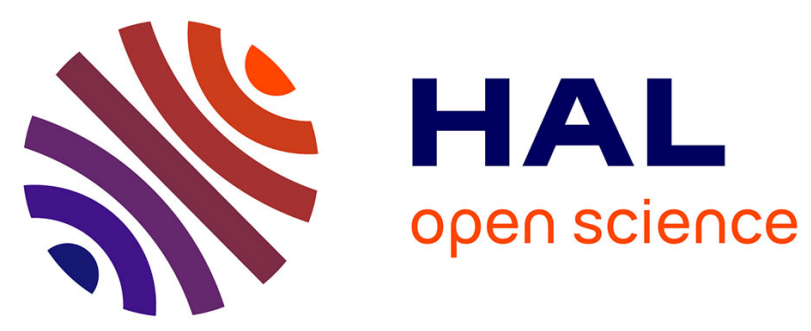

\title{
Wave propagation across a functionally graded interphase between soft and hard solids: Insight from a dynamic surface elasticity model
}

Ali Aghaei, Nicolas Bochud, Giuseppe Rosi, Salah Naili

\section{- To cite this version:}

Ali Aghaei, Nicolas Bochud, Giuseppe Rosi, Salah Naili. Wave propagation across a functionally graded interphase between soft and hard solids: Insight from a dynamic surface elasticity model. Journal of the Mechanics and Physics of Solids, 2021, 151, pp.104380. 10.1016/j.jmps.2021.104380 . hal-03209226

\section{HAL Id: hal-03209226 \\ https://hal.science/hal-03209226}

Submitted on 27 Apr 2021

HAL is a multi-disciplinary open access archive for the deposit and dissemination of scientific research documents, whether they are published or not. The documents may come from teaching and research institutions in France or abroad, or from public or private research centers.
L'archive ouverte pluridisciplinaire HAL, est destinée au dépôt et à la diffusion de documents scientifiques de niveau recherche, publiés ou non, émanant des établissements d'enseignement et de recherche français ou étrangers, des laboratoires publics ou privés. 
1. Wave propagation across a functionally graded interphase between soft and hard solids: Insight from a dynamic surface elasticity model

\author{
Ali Aghaei ${ }^{\mathrm{a}}$, Nicolas Bochud ${ }^{\mathrm{a}}$, Giuseppe Rosi $^{\mathrm{a}}$, Salah Naili ${ }^{\mathrm{a}}$ \\ ${ }^{a}$ MSME, CNRS UMR 8208, Univ Paris Est Creteil, Univ Gustave Eiffel, F-94010 Creteil, France
}

\begin{abstract}
Joining soft to hard materials is a challenging problem in modern engineering applications. In order to alleviate stress concentrations at the interface between materials with such a mismatch in mechanical properties, the use of functionally graded interphases is becoming more widespread in the design of the new generation of engineered composite materials. However, current macroscale models that aim at mimicking the mechanical behavior of such complex systems generally fail in incorporating the impact of microstructural details across the interphase because of computational burden. In this paper we propose to replace the thin, but yet finite, functionally graded interphase by a zero-thickness interface. This is achieved by means of an original model developed in the framework of surface elasticity, which accounts for both the elastic and inertial behavior of the actual interphase. The performance of the proposed equivalent model is evaluated in the context of elastic wave propagation, by comparing the calculated reflection coefficient to that obtained using different baseline models. Numerical results show that our dynamic surface elasticity model provides an accurate approximation of the reference interphase model over a broad frequency range. We demonstrate application of this modeling approach for the characterization of the graded tissue system at the tendon-to-bone interphase, which fulfills the challenging task of integrating soft to hard tissues over a submillimeter-wide region.
\end{abstract}

Keywords: Functionally graded interphase, Equivalent interface model, Enriched surface elasticity, Tendon-to-bone attachment, Elastic waves

\title{
1. Introduction
}

From a mechanical viewpoint, when materials having a dissimilar mechanical nature (e.g., soft and hard) are attached together, they typically display highly non-uniform deformations upon loading, eventually leading to stress concentration at their abrupt interface, which in turn increase the failure probability. An appealing solution to improve the integration between soft and hard materials consists in designing functionally graded interphases,

${ }^{*}$ Corresponding author: nicolas.bochud@u-pec.fr 
which are typically conceived as multilayers whose composition, microstructure and material properties gradually vary in space, in order to reduce mechanical stresses [1]. Naturally present in the human body [2], graded materials represent a source of inspiration that offers technological solutions for general engineering purposes as well as for biomedical applications. This concerns for instance the skin, which is a complex multilayered system, where each layer has a specific and age-related biomechanical behavior [3], the cortex of long bones, which displays an increasing gradient of porosity from the periosteum to the endosteum that is, in turn, related to a gradual change in mechanical properties such as tensile strength and elasticity [4], and the so-called entheses, which are specialized interfacial regions of the musculoskeletal system that allow joining connective tissues, such as tendon, ligament or cartilage, to bone [5].

In particular, the tendon-to-bone interphase serves the challenging task of connecting two highly dissimilar tissues over a very small region, which is typically a few hundreds of micrometers wide (see Fig. 1a). This interphase has the remarkable ability to minimize stress concentration and related failure modes, with the possibility to withstand forces higher than the body weight for millions of loading cycles $[6,7]$. These outstanding features, achieved by means of finely tuned gradients in structure, composition and biomechanical properties at different length scales (see Fig. 1b) [7, 8], are currently playing a significant role in the design of bioinspired interphases [9-11]. To unlock those mechanisms, computational models

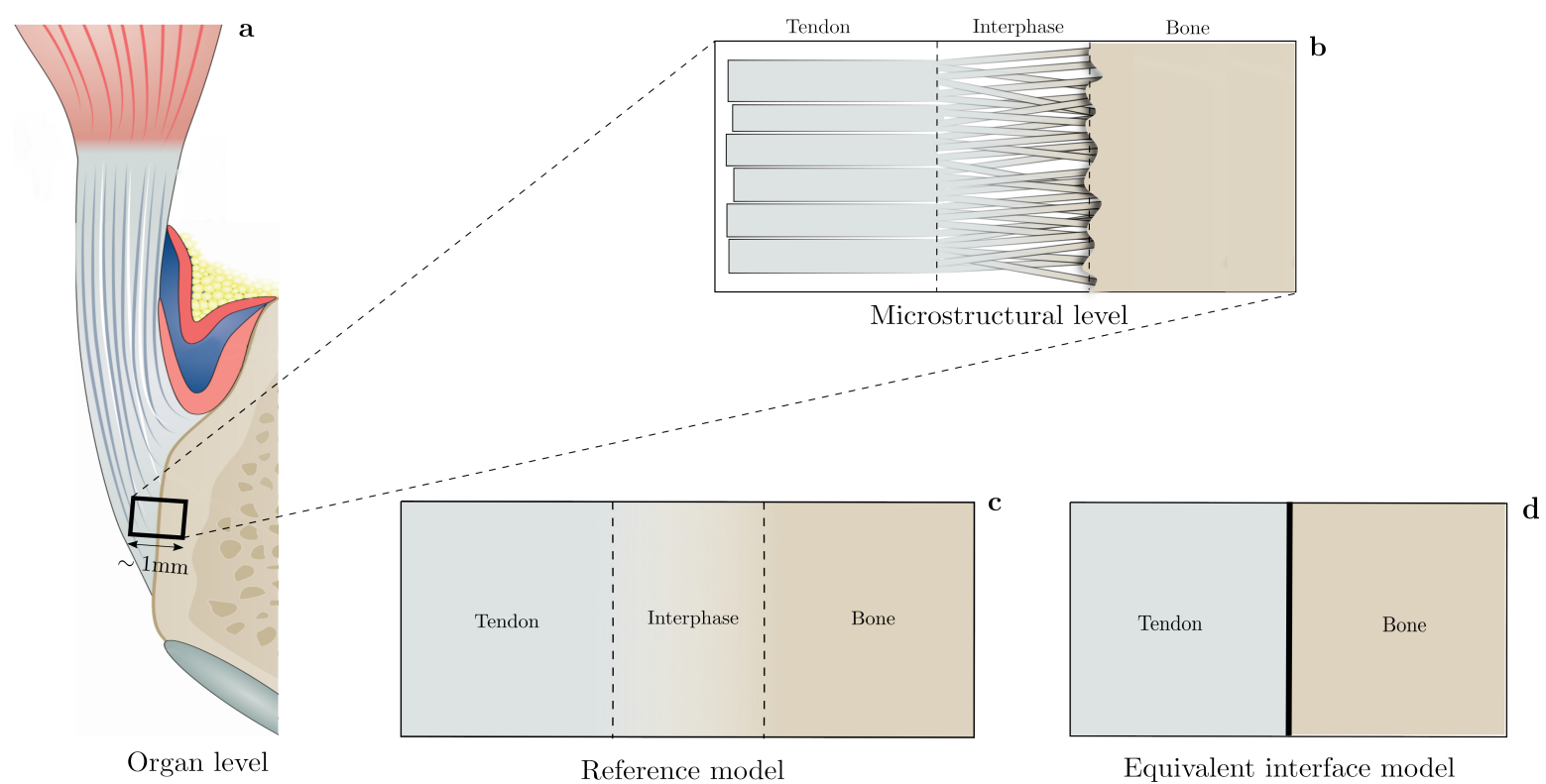

Figure 1: (a) Organ level illustration of the tendon-to-bone attachment at the insertion site corresponding to the Achilles tendon (image adapted from Ref. [12]); (b) Schematic of the attachment at the microstructural level, highlighting the gradients in composition and biomechanical properties across the tendon-to-bone interphase (image adapted from Ref. [8]); (c) Finite thickness interphase model with varying mechanical properties across the interphase; and (d) Equivalent interface model with specific properties.

were developed both to investigate fundamental anchoring strategies at the microstructural 
level [13-16] or to address applied orthopedic strategies at the organ level [17-19]. Notwithstanding, from a modeling viewpoint, it is highly challenging to bridge the gap between these two levels [20], and current models targeting reattachment procedures of the tendon-to-bone interphase should be enriched by including a more detailed description of the microstructure $[21,22]$. Indeed, to adequately capture the mechanical behavior of a graded interphase layer, the optimal choice would be to consider its exact geometry and varying mechanical properties across the two surrounding tissues [23]. However, this choice can be prohibitive when dealing with complex heterogeneous interphases. In particular, the finite size of such interphase, which is very small compared to that of the surrounding tissues, may cause computational burden when mesh refinements are required for convergence purposes. In contrast, a too basic model that would simplify the interphase to a large extent, or even ignore it, would fail in capturing its mechanics. To face these limitations, a possible solution consists in replacing the finite heterogeneous interphase (see Fig. 1c) by an equivalent interface with specific properties (see Fig. 1d) that retains the mechanical behavior of the original medium over a certain range of validity.

It is commonly accepted that such modeling approach can be satisfactorily addressed by enriching the equivalent model with additional fields [24]. Initial attempts proposed to tackle this problem by replacing the interphase by an interface with null thickness and purely elastic properties. A general framework for such general elastic interface model was comprehensively described in [25, 26], and was subsequently extended to account for the case where both the interphase and its surrounding media are anisotropic [27, 28] or the case of an elasto-plastic interphase [29, 30]. In the case of dynamic problems, however, and especially when dealing with wave propagation, a purely elastic surface model generally fails in accounting for the local interactions between the mechanical perturbation (e.g., a wave) and the interphase, even if its dimensions are much smaller than the involved mechanical characteristic length (e.g., the wavelength). Within this context, the inertial behavior of the interphase can have a considerable impact on its macroscopic dynamic response and should thus be included into the modeling strategy. This observation was also at the basis of the concept of a structural interface that possesses a finite thickness, which was introduced in [31] and further developed in [32], where the role of its inertial properties was highlighted. In the case of resonant meta-interfaces, an alternative approach was to obtain effective jump conditions by applying a suitable homogenization process [33, 34]. Moreover, some studies on elastic wave propagation showed that when the interphase is located between two surrounding media with microstructure, inertial properties also play a pivotal role in the modeling of the equivalent interface [35-37].

Following these recent findings, and in line with a former study by our group [38], we introduce here an enriched equivalent interface model, whose properties are defined by means of surface kinetic and potential energy densities. Furthermore, to account for the nonlinear gradients in mechanical properties across the interphase found at the tendon-to-bone attachement [39], we hypothesize that the displacement field can be approximated using a piece-wise affine profile, whose characteristics depend upon an additional degree-of-freedom within the interface. The performance of our modeling approach is evaluated by calculating the frequency-dependent reflection coefficient of a plane pressure wave under normal inci- 
dence. First, based on energetic concepts, the identification of the specific surface properties of the equivalent interface model is achieved by comparison with the reference interphase model. Second, an optimization procedure is conducted to investigate the impact of the additional degree-of-freedom. Third, the performance of the optimal equivalent model is compared to different models available in the literature, which typically serve as a baseline in finite element (FE) simulations at the organ scale. Our numerical results show that this enriched model with specific interface conditions provides a very accurate approximation of the reference model over a broad frequency range, thus outperforming more simplistic models that fail in capturing the complex dynamics of the interphase. As a by-product, the link between the position of the additional degree-of-freedom and the microstructural features of the interphase (e.g., competing gradients in mineral content and collagen fibers organization) is also discussed, thus opening promising perspectives for characterizing the tendon-to-bone attachment status. Elastic waves indeed represent a relevant nondestructive means to probe the interphase quality, as they possess intrinsic sensitivity to the mechanical properties contributing to the tendon-to-bone attachment strength. Overall this modeling approach represents the first building block for developing more sophisticated models targeting reattachment procedures at the organ scale that incorporate a more detailed description of properties at lower length scales.

The paper is structured as follows: Sect. 2 introduces the theoretical fundamentals of our modeling approach. The numerical results are then presented in Sect. 3. Finally, the strengths and limitations of the proposed model are discussed in Sect. 4.

\section{Theory}

Based on the variational principles [40-42], this section first presents the governing equations and boundary conditions for the two models depicted in Fig. 1c-d: (1) A reference model, which consists in a finite thickness interphase with a gradient in mechanical properties, surrounded by two homogeneous media (see Subsect. 2.1), and (2) an equivalent model in which the interphase is replaced by specific interface conditions between the two homogeneous media (see Subsect. 2.2). This general framework is then reduced to the specific case of a pressure plane wave propagating under normal incidence (see Subsect. 2.3). Finally, the strategy to identify the coefficients of the equivalent interface model is described in Subsect. 2.4.

\subsection{Reference interphase model}

Let us consider the Cartesian frame of reference with coordinates $\mathbf{R}\left(O ; \mathbf{e}_{1}, \mathbf{e}_{2}, \mathbf{e}_{3}\right)$, where $O$ is the origin and $\left(\mathbf{e}_{1}, \mathbf{e}_{2}, \mathbf{e}_{3}\right)$ is an orthonormal basis for the space. The coordinates of a point $M$ in $\mathbf{R}$ are specified by $\left(x_{1}, x_{2}, x_{3}\right)$ and the time is denoted by $t$. As depicted in Fig. 2, the reference model consists of two homogeneous half-spaces $\Omega^{-}=\left\{\left(x_{1}, x_{2}, x_{3}\right) \mid x_{2}, x_{3} \in\right.$ $\mathbb{R}$ and $\left.x_{1}<-\frac{h}{2}\right\}$ and $\Omega^{+}=\left\{\left(x_{1}, x_{2}, x_{3}\right) \mid x_{2}, x_{3} \in \mathbb{R}\right.$ and $\left.x_{1}>\frac{h}{2}\right\}$, separated by an heterogeneous interphase layer of thickness $h$, namely $\Omega^{I}=\left\{\left(x_{1}, x_{2}, x_{3}\right) \mid x_{2}, x_{3} \in \mathbb{R}\right.$ and $\left.\frac{h}{2}<x_{1}<\frac{h}{2}\right\}$, where $\mathbb{R}$ is the set of real numbers. In what follows, the equations of motion 


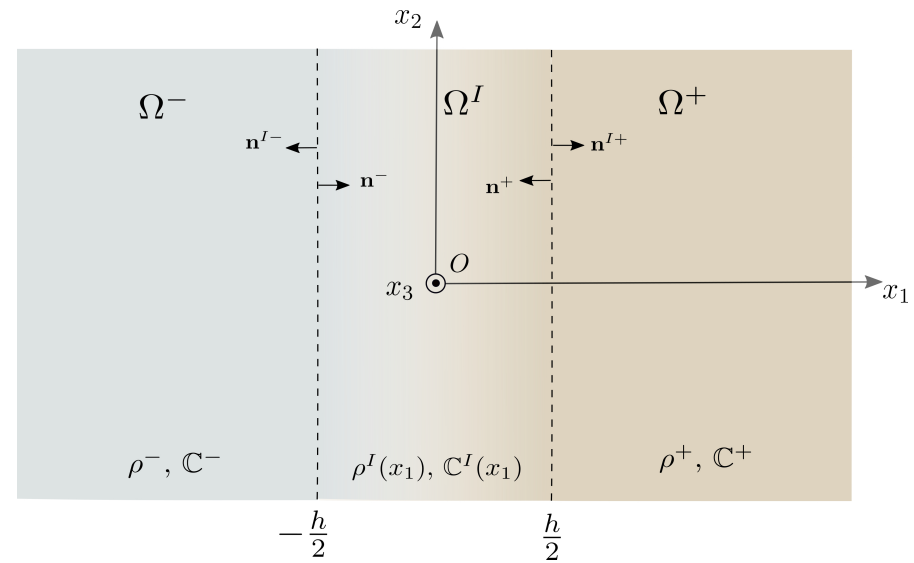

Figure 2: Modeling configuration of the reference model: A finite thickness interphase $\Omega^{I}$ with varying mechanical properties along the $x_{1}$-direction is surrounded by two homogeneous half-spaces $\Omega^{ \pm}$. Superscripts ,+- and $I$ denote the variables associated with the domains $\Omega^{+}, \Omega^{-}$, and $\Omega^{I}$, respectively.

are derived according to the least action principle. To this end, we introduce the action functional $\mathcal{A}^{\text {ref }}$ of the considered system as in [36],

$$
\mathcal{A}^{\mathrm{ref}}=\int_{\Omega \times\left(0, t_{f}\right)}\left[T^{+}+T^{-}+T^{I}-U^{+}-U^{-}-U^{I}\right] d A
$$

where $\Omega=\Omega^{ \pm} \cup \Omega^{I}$ spans the complete domain of interest, $\left(0, t_{f}\right)$ is a time interval and $d A$ represents the differential volume element. The kinetic and potential energy densities per unit volume, $T$ and $U$, associated with each domain of interest are defined as,

$$
\begin{aligned}
& T^{ \pm}=\frac{1}{2} \rho^{ \pm} \dot{\mathbf{u}}^{ \pm} \cdot \dot{\mathbf{u}}^{ \pm} \quad \text { and } \quad U^{ \pm}=\frac{1}{2} \boldsymbol{\sigma}^{ \pm}: \boldsymbol{\epsilon}^{ \pm}, \\
& T^{I}=\frac{1}{2} \rho^{I}\left(x_{1}\right) \dot{\mathbf{u}}^{I} \cdot \dot{\mathbf{u}}^{I} \quad \text { and } \quad U^{I}=\frac{1}{2} \boldsymbol{\sigma}^{I}: \boldsymbol{\epsilon}^{I},
\end{aligned}
$$

where $\rho^{ \pm}$are the mass densities of the domains $\Omega^{ \pm}$, and $\rho^{I}\left(x_{1}\right)$ is the mass density of the interphase $\Omega^{I}$, which varies along the $x_{1}$-direction. The displacement vector associated with each domain is denoted by $\mathbf{u}$, where the superimposed dot denotes the first derivative with respect to time. The stress and infinitesimal strain tensors associated with each domain are denoted by $\boldsymbol{\sigma}$ and $\boldsymbol{\epsilon}$, respectively, the latter being defined as $\boldsymbol{\epsilon}=(1 / 2)\left(\nabla \mathbf{u}+(\nabla \mathbf{u})^{T}\right)$, whereby $\nabla$ and the superscript $T$ denote the gradient and transpose operators, respectively. The operators dot (.) and colon (:) denote the scalar product of two vectors and the double contracted product of two tensors, respectively.

The least action principle implies that the variation of the action defined by Eq. (1) should verify that $\delta \mathcal{A}^{\text {ref }}=0$. This condition, along with the consideration of kinematic constraints on the boundaries, i.e., the continuity of displacements between the domains $\Omega^{ \pm}$ 
and $\Omega^{I}$, leads to the following boundary value problem for the reference model,

$$
\left\{\begin{array}{ll}
\nabla . \boldsymbol{\sigma}^{ \pm}-\rho^{ \pm} \ddot{\mathbf{u}}^{ \pm}=\mathbf{0} & \forall M \in \Omega^{ \pm} \\
\nabla . \boldsymbol{\sigma}^{I}-\rho^{I}\left(x_{1}\right) \ddot{\mathbf{u}}^{I}=\mathbf{0} & \forall M \in \Omega^{I} \\
\boldsymbol{\sigma}^{-} \mathbf{n}^{-}+\boldsymbol{\sigma}^{I} \mathbf{n}^{I-}=\mathbf{0} \\
\mathbf{u}^{-}-\mathbf{u}^{I}=\mathbf{0} \\
\boldsymbol{\sigma}^{I} \mathbf{n}^{I+}+\boldsymbol{\sigma}^{+} \mathbf{n}^{+}=\mathbf{0} \\
\mathbf{u}^{I}-\mathbf{u}^{+}=\mathbf{0}
\end{array}\right\} \text { for } \quad x_{1}=-\frac{h}{2},
$$

where $\nabla$. denote the divergence operator. The constitutive relation associated with each domain is defined in the frame of the linear elasticity, i.e., $\boldsymbol{\sigma}^{ \pm}=\mathbb{C}^{ \pm} \boldsymbol{\epsilon}^{ \pm}$and $\boldsymbol{\sigma}^{I}=\mathbb{C}^{I}\left(x_{1}\right) \boldsymbol{\epsilon}^{I}$, whereby $\mathbb{C}^{ \pm}$is the fourth-order elasticity tensor of the domains $\Omega^{ \pm}$, and $\mathbb{C}^{I}\left(x_{1}\right)$ is the fourthorder elasticity tensor of the interphase $\Omega^{I}$, which varies with respect to the $x_{1}$-direction.

\subsection{Equivalent interface model}

This section introduces the equivalent model based on surface elasticity, in which the finite thickness heterogeneous interphase is replaced by specific interface conditions. To this end, kinetic and potential energy densities of the interphase, $T^{I}$ and $U^{I}$ from Eq. (3), are now substituted by surface energy densities. The action functional $\mathcal{A}$ for the equivalent system can thus be defined in a similar manner as in Sect. 2.1,

$$
\mathcal{A}=\int_{\Omega \times\left(0, t_{f}\right)}\left[T^{+}+T^{-}-U^{+}-U^{-}\right] d A+\int_{\partial \Omega \times\left(0, t_{f}\right)}\left[T^{S}-U^{S}\right] d S,
$$

where $\partial \Omega$ is the mid-surface of the domain $\Omega^{I}$ and $d S$ represents the differential surface element, so that

$$
T^{S}=\int_{-\frac{h}{2}}^{x_{l}} T^{I} d x_{1}+\int_{x_{l}}^{\frac{h}{2}} T^{I} d x_{1} \quad \text { and } \quad U^{S}=\int_{-\frac{h}{2}}^{x_{l}} U^{I} d x_{1}+\int_{x_{l}}^{\frac{h}{2}} U^{I} d x_{1},
$$

where $-\frac{h}{2}<x_{l}<\frac{h}{2}$. Owing to the continuity of displacements at the boundaries, we can state that $\mathbf{u}^{I}\left(-\frac{h}{2}\right)=\mathbf{u}^{-}\left(-\frac{h}{2}\right)$ and $\mathbf{u}^{I}\left(\frac{h}{2}\right)=\mathbf{u}^{+}\left(\frac{h}{2}\right)$, where, for the sake of conciseness, the dependence of the fields on the spatial coordinates $x_{2}$ and $x_{3}$, as well as on time $t$, is dropped. We furthermore assume that the integrals in Eq. (6) are quadratic forms of the displacement and velocity fields evaluated on the $x_{2} x_{3}$-plane for $x_{1}$ equal to $-\frac{h}{2}, x_{l}$ or $\frac{h}{2}$. Altogether, these hypotheses allow deriving a general form for the surface energy densities as,

$$
\begin{aligned}
T^{S} & =\frac{1}{2}\left[\boldsymbol{m}^{+} \dot{\mathbf{u}}^{+} \cdot \dot{\mathbf{u}}^{+}+\boldsymbol{m}_{l} \dot{\mathbf{u}}_{l} \cdot \dot{\mathbf{u}}_{l}+\boldsymbol{m}^{-} \dot{\mathbf{u}}^{-} \cdot \dot{\mathbf{u}}^{-}+2 \boldsymbol{\gamma}_{1} \dot{\mathbf{u}}^{-} \cdot \dot{\mathbf{u}}_{l}+2 \boldsymbol{\gamma}_{2} \dot{\mathbf{u}}_{l} \cdot \dot{\mathbf{u}}^{+}\right], \\
U^{S} & =\frac{1}{2} \boldsymbol{K}_{1}\left[\mathbf{u}_{l}-\mathbf{u}^{-}\right] \cdot\left[\mathbf{u}_{l}-\mathbf{u}^{-}\right]+\frac{1}{2} \boldsymbol{K}_{2}\left[\mathbf{u}^{+}-\mathbf{u}_{l}\right] \cdot\left[\mathbf{u}^{+}-\mathbf{u}_{l}\right],
\end{aligned}
$$

where $\mathbf{u}_{l}=\mathbf{u}^{I}\left(x_{l}\right)$. Note that, by abuse of notation, we dropped the dependence on space for all displacements. These surface energy densities, which are concentrated in the mid-surface 
$\partial \Omega$ between the domains $\Omega^{+}$and $\Omega^{-}$, can be interpreted by means of the generalized springmass system depicted in Fig. 3. Within this frame, the second-order tensors $\boldsymbol{m}^{ \pm}$and $\boldsymbol{m}_{l}$ represent masses concentrated at both sides of the interface and at position $x_{l}$, respectively; the second-order tensors $\boldsymbol{K}_{1}$ and $\boldsymbol{K}_{2}$ represent surface stiffnesses; and finally the secondorder tensors $\gamma_{1}$ and $\gamma_{2}$ account for the kinetic interactions between the displacement fields.

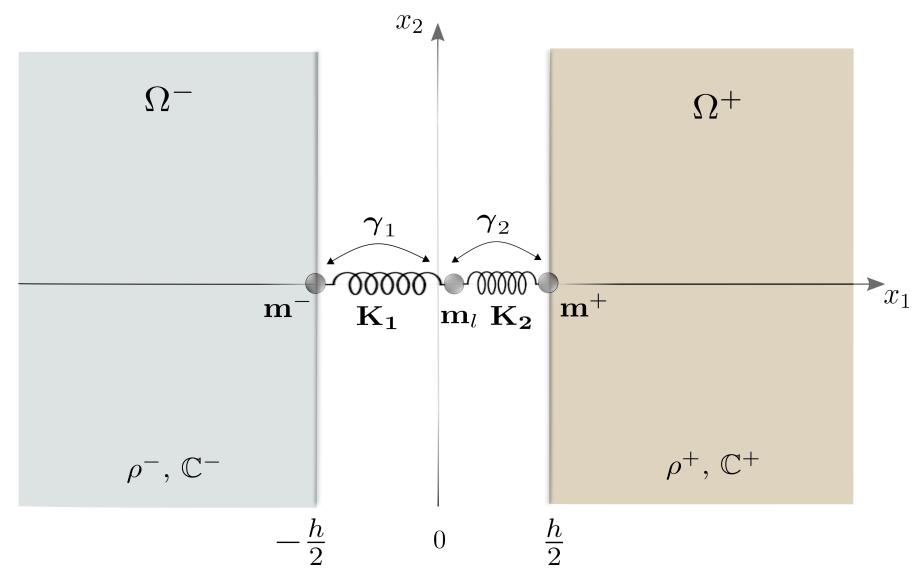

Figure 3: Modeling configuration of the equivalent model with specific interface conditions. The generalized spring-mass system illustrates the role played by the additional degree-of-freedom $l$ to account for the dynamic interactions across the interphase.

It is worth pointing out that, in the most general case, all these constitutive tensors are function of space and time (or frequency in the harmonic regime). Nevertheless, for the model to be exploitable, i.e., to link these tensors to the physical characteristics of the interface rather than to its dynamic properties, a possibility is to turn them into constants. Here the calculation of these tensors will be achieved by selecting specific profiles for the displacement and velocity fields across the interphase, $\mathbf{u}^{I}\left(x_{1}\right)$ and $\dot{\mathbf{u}}^{I}\left(x_{1}\right)$, which fulfill the aforementioned constraints at the location $x_{1}$ given by the values $-\frac{h}{2}, x_{l}$ or $\frac{h}{2}$. The natural consequence of this choice is that all surface energy terms will be considered as approximations only.

Again, the variation of the action $\delta \mathcal{A}$ should verify that $\delta \mathcal{A}=0$, thus leading to the following boundary value problem,

$$
\left\{\begin{array}{l}
\nabla . \boldsymbol{\sigma}^{ \pm}-\rho^{ \pm} \ddot{\mathbf{u}}^{ \pm}=\mathbf{0}, \quad \forall M \in \Omega^{ \pm} \\
\boldsymbol{\sigma}^{-} \mathbf{n}^{-}-\boldsymbol{K}_{1}\left(\mathbf{u}_{l}-\mathbf{u}^{-}\right)+\boldsymbol{m}^{-} \ddot{\mathbf{u}}^{-}+\gamma_{1} \ddot{\mathbf{u}}_{l}=\mathbf{0} \\
\boldsymbol{\sigma}^{+} \mathbf{n}^{+}+\boldsymbol{K}_{2}\left(\mathbf{u}^{+}-\mathbf{u}_{l}\right)+\boldsymbol{m}^{+} \ddot{\mathbf{u}}^{+}+\gamma_{2} \ddot{\mathbf{u}}_{l}=\mathbf{0} \\
\boldsymbol{K}_{1}\left(\mathbf{u}_{l}-\mathbf{u}^{-}\right)-\boldsymbol{K}_{2}\left(\mathbf{u}^{+}-\mathbf{u}_{l}\right)+\boldsymbol{m}_{l} \ddot{\mathbf{u}}_{l}+\gamma_{1} \ddot{\mathbf{u}}^{-}+\gamma_{2} \ddot{\mathbf{u}}^{+}=\mathbf{0}
\end{array}\right.
$$

where the first equation retains the same form than that of Eq. (4), whereas the other three now account for the specific interface conditions, in which, by abuse of notation, the stress tensors are now stated as $\boldsymbol{\sigma}^{-}=\boldsymbol{\sigma}^{I}\left(-\frac{h}{2}\right)$ and $\boldsymbol{\sigma}^{+}=\boldsymbol{\sigma}^{I}\left(\frac{h}{2}\right)$. It should be noted that this 
boundary value problem could be easily implemented in a standard FE code, as it only involves a modification of the boundary conditions.

\subsection{Pressure plane wave propagation under normal incidence}

We now consider the problem of a plane elastic wave propagating under normal incidence across the considered functionally graded interphase. In this way, our modeling approach is reduced to an incident and a reflected longitudinal bulk wave in the domain $\Omega^{-}$and a transmitted longitudinal bulk wave in the domain $\Omega^{+}$. By assuming a general harmonic solution for the interphase $\Omega^{I}$, the general solution for a plane wave propagating along the $x_{1}$-direction reads as

$$
\begin{aligned}
& u^{-}\left(x_{1}, t\right)=\left(A_{i} \exp \left(j k_{p}^{-} x_{1}\right)+A_{r} \exp \left(-j k_{p}^{-} x_{1}\right)\right) \exp (-j \omega t) \\
& u^{+}\left(x_{1}, t\right)=A_{t} \exp \left(j\left(k_{p}^{+} x_{1}-\omega t\right)\right) \\
& u^{I}\left(x_{1}, t\right)=g\left(x_{1}\right) \exp (-j \omega t)
\end{aligned}
$$

where $\omega$ represents the angular frequency and $j$ is the unit imaginary number. The variable $k_{p}^{ \pm}=\omega / c_{p}^{ \pm}$denotes the wave number, which depends upon the longitudinal bulk wave velocity $c_{p}^{ \pm}$in the domain $\Omega^{ \pm}$. The variables $A_{i}, A_{r}$ and $A_{t}$ denote the amplitudes of the incident, reflected and transmitted plane waves, respectively. The function $g\left(x_{1}\right)$ represents the unknown amplitude of the harmonic solution in the interphase, which accounts for the material heterogeneity along the $x_{1}$-direction. Substituting the wave solutions from Eq. (10) into the boundary value problem from Eq. (4), and working through these analytical equations to remove the variables $A_{r}$ and $A_{t}$, yield the following boundary value problem for the function $g\left(x_{1}\right)$ in strong form,

$$
\left\{\begin{array}{l}
\left(C_{11}^{I}\left(x_{1}\right) g_{, 1}\left(x_{1}\right)\right)_{, 1}+\omega^{2} \rho^{I}\left(x_{1}\right) g\left(x_{1}\right)=0 \\
C_{11}^{I}\left(-\frac{h}{2}\right) g_{, 1}\left(-\frac{h}{2}\right)+j k_{p}^{-} C_{11}^{-} g\left(-\frac{h}{2}\right)-2 j k_{p}^{-} C_{11}^{-} A_{i} \exp \left(-j k_{p}^{-} \frac{h}{2}\right)=0 \\
C_{11}^{I}\left(\frac{h}{2}\right) g_{, 1}\left(\frac{h}{2}\right)-j k_{p}^{+} C_{11}^{+} g\left(\frac{h}{2}\right)=0
\end{array},\right.
$$

where the derivative with respect to $x_{1}$ is denoted by $\left(_{, 1}\right)$ and $C_{11}$ is the stiffness coefficient along the $x_{1}$-direction (in Voigt notation) associated with each domain. The first relation of Eq. (11) is an ordinary differential equation with respect to the coordinate $x_{1}$, whereas the two last relations account for the boundary conditions at positions $x_{1}=-\frac{h}{2}$ and $x_{1}=$ $\frac{h}{2}$, respectively. Note that such boundary value problem in strong form must be solved numerically.

In the same vein, the boundary value problem associated with the equivalent interface model (recall Eq. (9)) can be simplified as follows in the case of a pressure plane wave under 
normal incidence,

$$
\left\{\begin{array}{l}
C_{11}^{ \pm} u_{, 11}^{ \pm}-\rho^{ \pm} \ddot{u}^{ \pm}=0, \quad \forall M \in \Omega^{ \pm} \\
C_{11}^{-} u_{, 1}^{-}-K_{1}\left(u_{l}-u^{-}\right)+m^{-} \ddot{u}^{-}+\gamma_{1} \ddot{u}_{l}=0 \\
-C_{11}^{+} u_{, 1}^{+}+K_{2}\left(u^{+}-u_{l}\right)+m^{+} \ddot{u}^{+}+\gamma_{2} \ddot{u}_{l}=0 \\
K_{1}\left(u_{l}-u^{-}\right)-K_{2}\left(u^{+}-u_{l}\right)+m_{l} \ddot{u}_{l}+\gamma_{1} \ddot{u}^{-}+\gamma_{2} \ddot{u}^{+}=0
\end{array}\right.
$$

where it should be noted that the second-order tensors of the surface energy terms are now all reduced to scalar coefficients. Furthermore, we hypothesize that the general solution for the displacement in the interface can be stated as

$$
u_{l}(t)=B \exp (-j \omega t),
$$

where $B$ represents the unknown amplitude of the harmonic solution in the interface. Substituting Eq. (13), along with the wave solutions associated with the surrounding domains $\Omega^{ \pm}$from Eq. (10), into the boundary value problem from Eq. (12) leads to the following linear equation system

$$
\left(\begin{array}{ccc}
-j k_{p}^{-} C_{11}^{-}+K_{1}-\omega^{2} m^{-} & -K_{1}-\omega^{2} \gamma_{1} & 0 \\
0 & -K_{2}-\omega^{2} \gamma_{2} & -j k_{p}^{+} C_{11}^{+}+K_{2}-\omega^{2} m^{+} \\
-K_{1}-\omega^{2} \gamma_{1} & K_{1}+K_{2}-\omega^{2} m_{l} & -K_{2}-\omega^{2} \gamma_{2}
\end{array}\right)\left[\begin{array}{c}
A_{r} \\
B \\
A_{t}
\end{array}\right]=\left(\begin{array}{c}
-\left(j k_{p}^{-} C_{11}^{-}+K_{1}-\omega^{2} m^{-}\right) A_{i} \\
0 \\
\left(K_{1}+\omega^{2} \gamma_{1}\right) A_{i}
\end{array}\right),
$$

whose solution allows determining the unknown amplitudes, $A_{r}$ and $A_{t}$, of the reflected and transmitted waveforms. Note that, in contrast to the boundary value problem associated with the reference interphase model (recall Eq. (11)), the resulting system for the equivalent interface can be solved analytically.

\subsection{Identification of the equivalent model coefficients}

This section presents the adopted strategy for identifying the coefficients of the surface kinetic and internal energy densities, i.e., $m^{-}, m_{l}, m^{+}, \gamma_{1}, \gamma_{2}, K_{1}$, and $K_{2}$. Towards this goal, we hypothesize that the displacement field is directed towards the surface normal, i.e., $\mathbf{u}^{I}=u^{I}\left(x_{1}\right) \mathbf{e}_{1}$, and that it can be approximated using a piece-wise affine profile with respect to the coordinate $x_{1}$ (see Fig. 4 ).

In such a case, the kinetic and potential energy densities across the interphase (recall Eq. (3)) can be written as

$$
T^{I}=\frac{1}{2} \int_{-\frac{h}{2}}^{\frac{h}{2}} \rho^{I}\left(x_{1}\right)\left(v^{I}\left(x_{1}\right)\right)^{2} d x_{1} \quad \text { and } \quad U^{I}=\frac{1}{2} \int_{-\frac{h}{2}}^{\frac{h}{2}} C_{11}^{I}\left(x_{1}\right)\left(u_{, 1}^{I}\left(x_{1}\right)\right)^{2} d x_{1},
$$

where

$$
u^{I}\left(x_{1}\right)=\left\{\begin{array}{ll}
\left(\frac{2\left(u_{l}-u^{-}\right)}{h+2 x_{l}}\right) x_{1}+\left(\frac{u_{l} h+2 u^{-} x_{l}}{h+2 x_{l}}\right) \quad \text { for } \quad-\frac{h}{2}<x_{1}<x_{l} \\
\left(\frac{2\left(u^{+}-u_{l}\right)}{h-2 x_{l}}\right) x_{1}-\left(\frac{2 u^{+} x_{l}-u_{l} h}{h-2 x_{l}}\right) \quad \text { for } \quad x_{l}<x_{1}<\frac{h}{2}
\end{array},\right.
$$




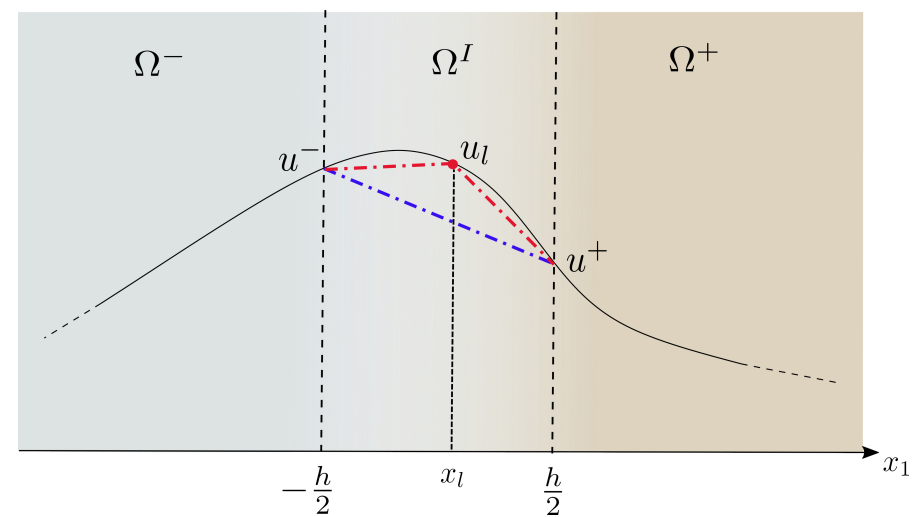

Figure 4: Illustration of the displacement field across the interphase for an arbitrarily selected frequency: Comparison between the exact profile (continuous black line) used in the reference model and the piece-wise affine profile (dashed red line) used in the equivalent interface model. The affine profile (dashed blue line) proposed in [38] is shown for comparison.

in which $u^{-}, u_{l}$ and $u^{+}$are the values of the displacement field $u^{I}\left(x_{1}\right)$ at the location $x_{1}$ given by the values $-\frac{h}{2}, x_{l}$ or $\frac{h}{2}$. Note that, since we assumed a general harmonic solution for the displacement field in the interphase, the velocity field $v^{I}\left(x_{1}\right)$ will have the same piece-wise form, modulo the constant complex factor $j \omega$, than that of Eq. (16), with $v^{-}, v_{l}$ and $v^{+}$being the values of the velocity field at the location $x_{1}$ given by the values $-\frac{h}{2}, x_{l}$ or $\frac{h}{2}$. According to the pressure plane wave assumption, the kinetic and potential energies per unit surface, $T^{S}$ and $U^{S}$, can now be written as

$$
\begin{aligned}
T^{S} & =\frac{1}{2}\left[m^{+}\left(v^{+}\right)^{2}+m_{l}\left(v_{l}\right)^{2}+m^{-}\left(v^{-}\right)^{2}+2 \gamma_{1} v^{-} v_{l}+2 \gamma_{2} v_{l} v^{+}\right], \\
U^{S} & =\frac{1}{2} K_{1}\left(u_{l}-u^{-}\right)^{2}+\frac{1}{2} K_{2}\left(u^{+}-u_{l}\right)^{2},
\end{aligned}
$$

where the necessary conditions for the kinetic energy density, $T^{S}$, to be a positive definite quadratic form are that $m^{ \pm}>0, \gamma_{2}^{2}<\frac{1}{2} m^{+} m_{l}$, and $\gamma_{1}^{2}<\frac{1}{2} m^{-} m_{l}$. By inserting the displacement and velocity fields, $u^{I}\left(x_{1}\right)$ and $v^{I}\left(x_{1}\right)$, into Eq. (15), the outcome can then be compared to Eq. (17) by making use of the relations defined in Eq. (6), thus allowing for the identification of the coefficients belonging to the surface kinetic and potential energy 
densities $T^{S}$ and $U^{S}$ as

$$
\begin{aligned}
& m^{-}=4 \int_{-\frac{h}{2}}^{x_{l}} \rho_{I}\left(x_{1}\right)\left(\frac{x_{1}-x_{l}}{h+2 x_{l}}\right)^{2} d x_{1} \\
& m^{+}=4 \int_{x_{l}}^{\frac{h}{2}} \rho_{I}\left(x_{1}\right)\left(\frac{x_{1}-x_{l}}{h-2 x_{l}}\right)^{2} d x_{1} \\
& m_{l}=\int_{-\frac{h}{2}}^{x_{l}} \rho_{I}\left(x_{1}\right)\left(\frac{h+2 x_{1}}{h+2 x_{l}}\right)^{2} d x_{1}+\int_{x_{l}}^{\frac{h}{2}} \rho_{I}\left(x_{1}\right)\left(\frac{h-2 x_{1}}{h-2 x_{l}}\right)^{2} d x_{1} \\
& \gamma_{1}=2 \int_{-\frac{h}{2}}^{x_{l}} \rho_{I}\left(x_{1}\right) \frac{\left(h+2 x_{1}\right)\left(-x_{1}+x_{l}\right)}{\left(h+2 x_{l}\right)^{2}} d x_{1} \\
& \gamma_{2}=2 \int_{x_{l}}^{\frac{h}{2}} \rho_{I}\left(x_{1}\right) \frac{\left(h-2 x_{1}\right)\left(x_{1}-x_{l}\right)}{\left(h-2 x_{l}\right)^{2}} d x_{1} \\
& K_{1}=\frac{1}{x_{l}^{2}} \int_{-\frac{h}{2}}^{x_{l}} C_{11}^{I}\left(x_{1}\right) d x_{1} \\
& K_{2}=\frac{1}{\left(h-x_{l}\right)^{2}} \int_{x_{l}}^{\frac{h}{2}} C_{11}^{I}\left(x_{1}\right) d x_{1} .
\end{aligned}
$$

In our earlier study [38], the equivalent interface model was associated with an affine displacement field (dashed blue line in Fig. 4). To serve as a comparison with Eqs. (17)-(18), the related expressions for the kinetic and potential energy densities per unit surface, along with the identified coefficients, are briefly recalled in Appendix A.

\section{Numerical results}

The performance of the proposed enriched model is assessed by evaluating the frequencydependent reflection coefficient in a reflection/transmission problem between two homogeneous solid half-spaces, which are separated by a finite thickness and heterogeneous interphase. First, we identify the specific surface properties of the equivalent interface by comparison with the reference interphase. Second, we perform an optimization procedure to investigate the impact of the position $x_{l}$ of the additional degree-of-freedom on the modeling performance. The optimal equivalent model is subsequently compared to different baselines.

Towards these goals, we consider here an interphase whose stiffness and density vary along the $x_{1}$-direction. This configuration is inspired by our recent multiscale modeling results [39], which showed that the tendon-to-bone interphase can be seen as a continuous functionally graded material. The selected model parameters for the two surroundings tissues, i.e., tendon $\Omega^{-}$and bone $\Omega^{+}$, together with the finite thickness $h$ of the interphase, are summarized in Tab. 1.

The gradients in mechanical properties across the interphase $\Omega^{I}$, which are used as further input in the reference model, are depicted in Fig. 5. The effective stiffness variation at the mesoscale is the result of competing gradients in mineral content and collagen fibers organization at lower length scales. Indeed, it has been shown that the linear increase in 


\begin{tabular}{|c|c|c|c|c|}
\hline \multicolumn{2}{|c|}{ Mass density $\left(\mathrm{g} \cdot \mathrm{cm}^{-3}\right)$} & \multicolumn{2}{|c|}{ Stiffness coefficient (GPa) } & \multirow{2}{*}{$\begin{array}{c}\text { Thickness }(\mu \mathrm{m}) \\
h\end{array}$} \\
\hline$\rho^{-}$ & $\rho^{+}$ & $C_{11}^{-}$ & $C_{11}^{+}$ & \\
\hline 1.22 & 1.88 & 4.37 & 27.48 & 300 \\
\hline
\end{tabular}

Table 1: Model parameters for the two surroundings tissues $\Omega^{ \pm}$and the interphase $\Omega^{I}$.

mineral content caused a stiffening of the interphase that became significant beyond a certain percolation threshold (approximately located between $0-50 \mu \mathrm{m}$ in Fig. 5a), whereas the decreasing collagen fiber organization across the interphase led to a reduced tissue stiffness along the main fibers direction $[14,39]$. In contrast, the effective mass density evolves linearly between the two surrounding tissues (see Fig. 5b).
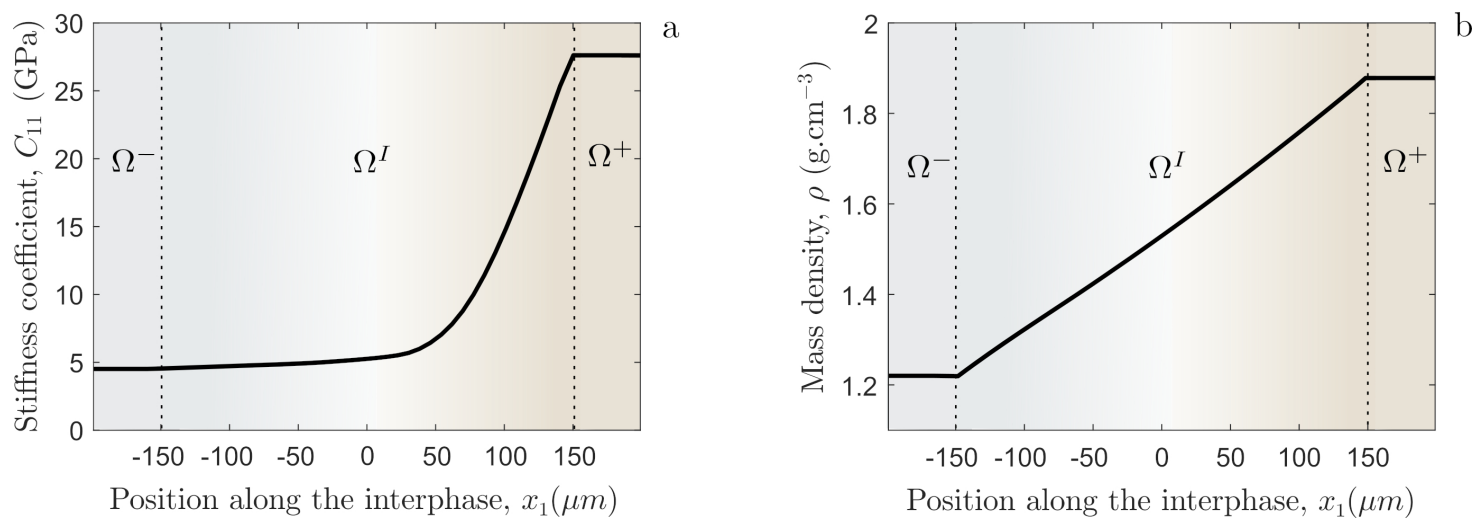

Figure 5: Graded mechanical properties across the tendon-to-bone interphase: (a) effective stiffness coefficient $C_{11}$ and (b) effective mass density $\rho$. These trends are based upon the multiscale model reported in $[39]$.

\subsection{Evaluation of the equivalent interface model}

To validate the proposed equivalent interface model, we first evaluate the reflection coefficient $R=\left|A_{r} / A_{i}\right|^{2}$ as a function of frequency, in which the amplitude $A_{i}$ of the incident plane wave was set to unity. For a better interpretation, the reflection coefficient $R$ was calculated as well as a function of the ratio between the average wavelength $\bar{\lambda}$ and the thickness $h$ of the interphase. The average wavelength $\bar{\lambda}$ was defined as

$$
\bar{\lambda}=\frac{\bar{c}_{p}^{I}}{f}, \quad \text { with } \bar{c}_{p}^{I}=\sqrt{\frac{\bar{C}_{11}^{I}}{\bar{\rho}^{I}}}
$$

where $\bar{c}_{p}^{I}, \bar{C}_{11}^{I}$ and $\bar{\rho}^{I}$ are the average phase velocity, the average stiffness and the average mass density, respectively. 
The reference model from Eq. (11) is solved using a standard FE code such as Comsol Multiphysics ${ }^{\circledR}$, from which the reflection coefficient, $R^{\text {ref }}$ can be derived as

$$
R^{\mathrm{ref}}=\left|\frac{A_{r}}{A_{i}}\right|^{2}=\left|\frac{g\left(-\frac{h}{2}\right)-A_{i} \exp \left(-j k_{p}^{-} \frac{h}{2}\right)}{A_{i} \exp \left(j k_{p}^{-} \frac{h}{2}\right)}\right|^{2} .
$$

In contrast, the reflection coefficient associated with the equivalent interface model can be calculated analytically using Eq. (14). Note, however, that this enriched modeling approach now depends upon the position of the additional degree-of-freedom $x_{l}$, whose optimal location is a priori not known. To serve as an example, the coefficients of the kinetic and potential energy densities were first calculated for two arbitrarily selected values of $x_{l}$ using Eq. (18). The numerical results are depicted in Fig. 6, in which the proposed model (dashed red line) with different values of $x_{l}$ is compared to the reference interphase model (continuous black line), to a homogeneous interphase $\Omega^{I}$ with averaged mechanical properties (dashed gray line), and to an abrupt transition (continuous gray line) corresponding to the case where the surroundings tissues $\Omega^{ \pm}$are placed directly in contact. As can be observed in

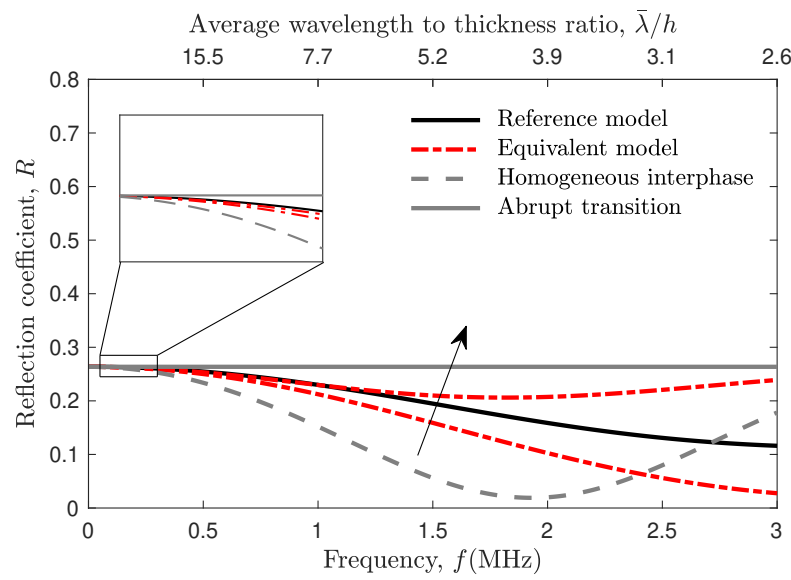

Figure 6: Frequency-dependent reflection coefficient $R$ obtained using: (1) the reference model (continuous black line); (2) a homogeneous interphase (dashed gray line); (3) an abrupt transition (continuous gray line); and (4) the equivalent interface model (dashed red lines) for two arbitrarily selected values of $x_{l}$ equal to -50 and $50 \mu \mathrm{m}$. The black arrow indicates the resulting trend with respect to increasing values of $x_{l}$.

the enlargement of Fig. 6 , for a low frequency regime below $0.3 \mathrm{MHz}$ (i.e., high ratio $\bar{\lambda} / h$ ), the equivalent interface model satisfactorily captures the dynamics of the reference model, independently of the position of the additional degree-of-freedom $x_{l}$. At an intermediate frequency regime around $0.3-2.5 \mathrm{MHz}$, the choice of $x_{l}$ has a significant impact on the ability of our model to match the reference one, and thus account for the gradient in mechanical properties, which in turn reflect microstructural features at lower length scales. As expected, when the ratio of the average wavelength to the interphase thickness decreases towards values below 3 , the deviation from the reference model increases, and our approximated model 
fails in capturing the complex dynamics of the interphase, which is associated with stronger wave-interphase interactions. Nonetheless, it is worth mentioning that, overall, the deviation of this model from the reference ones is much lower than that obtained using a simple abrupt transition or a homogeneous interphase with averaged mechanical properties, which represent the commonly used baselines in FE simulations at the organ scale [43-45].

\subsection{Optimization of the equivalent interface model}

Second, an optimization procedure was conducted to further investigate the impact of the additional degree-of-freedom position $x_{l}$ on the performance of the proposed model. To this end, the relative cumulative error on the reflection coefficient (denoted by err) between the one of the reference model (denoted by $R^{\text {ref}}$ ) and the one of the equivalent interface model (denoted by $R$ ) was calculated by sweeping the frequency and the position of $x_{l}$ across the interphase as

$$
\operatorname{err}\left(x_{l}, f\right)=\frac{1}{N_{\max }} \sum_{n=1}^{N_{\max }}\left(\frac{R^{\mathrm{ref}}\left(f_{n}\right)-R\left(x_{l}, f_{n}\right)}{R^{\operatorname{ref}}\left(f_{n}\right)}\right)^{2},
$$

where $N_{\max }$ is an integer value corresponding to the maximal frequency position. Figure $7 \mathrm{a}$ represents the dynamics of the error as a function of the position of $x_{l}$ and the frequency $f$. As can be observed, at a low frequency regime below $0.3 \mathrm{MHz}$, the equivalent interface model
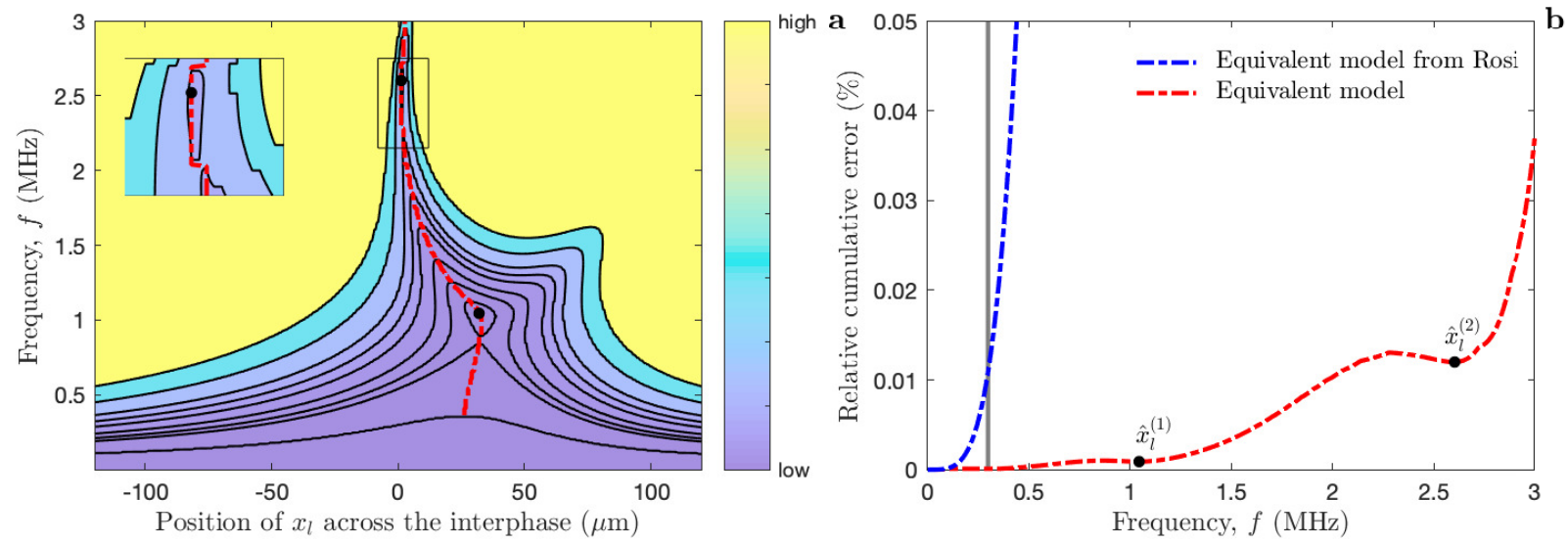

Figure 7: (a) Relative cumulative error on the reflection coefficient (Eq. (21)) between the one of the reference model and the one of the equivalent interface model as a function of the frequency and the position of the additional degree-of-freedom $x_{l}$ across the interphase. The dashed red line represents the optimal $x_{l}$-path as a function of the frequency, whereas the black dots depict the local minima $x_{l}^{(1)}$ and $x_{l}^{(2)}$. The region around the second local minima is displayed in the insert. (b) Relative cumulative error along the optimal $x_{l}$-path as a function of the frequency (dashed red line). The error between the reference model and the model presented in [38] is shown for comparison (dashed blue line). The gray line represents the frequency threshold $(0.3 \mathrm{MHz})$ below which the error is independent of the position $x_{l}$.

provides an accurate approximation of the reference model independently of the position of the additional degree-of-freedom $x_{l}$. Towards higher frequencies, the performance of the equivalent model slightly decreases, but there is a specific path along $x_{l}$ (displayed as a dashed red line) for which the error remains reasonably low. Interestingly, there are two 
local minima located at around 1 and $2.6 \mathrm{MHz}$ along this path (black dots), for which the optimal values of $\hat{x}_{l}$ are equal to 32 and $1.2 \mu \mathrm{m}$, respectively (i.e., thicknesses that fall within the range corresponding to the percolation threshold in Fig. 5a). A more advanced insight is provided in Fig. 7b, which depicts the relative cumulative error along the optimal $x_{l}$-path as a function of the frequency, thus clearly highlighting the presence of two local minima, i.e., $x_{l}^{(1)}$ and $x_{l}^{(2)}$. It is worth mentioning that the error associated with $x_{l}^{(2)}$ at $2.6 \mathrm{MHz}$ is of the same order than that obtained using the model from [38] at $0.3 \mathrm{MHz}$ (low frequency regime represented as a continuous gray line). Therefore, fine-tuning the position of the additional degree-of-freedom $x_{l}$ in our modeling approach allows enlarging nearly 8 times the validity range with respect to that model.

To further assess the performance of our enriched model, Fig. 8 now depicts the obtained frequency-dependent reflection coefficient $R$ for the optimal position $x_{l}^{(2)}$ (dashed red line), which is compared to the reference model (continuous black line), an abrupt transition (continuous gray line), the equivalent model from [38] (dashed blue line) and an equivalent model possessing only elastic properties (dotted red line), that is to say non-inertial interface conditions (i.e., $m^{+}=m^{-}=m_{l}=0 \mathrm{~kg} \cdot \mathrm{m}^{-2}$ and $\gamma_{1}=\gamma_{2}=0 \mathrm{~kg} . \mathrm{m}^{-2}$ ). As expected from the

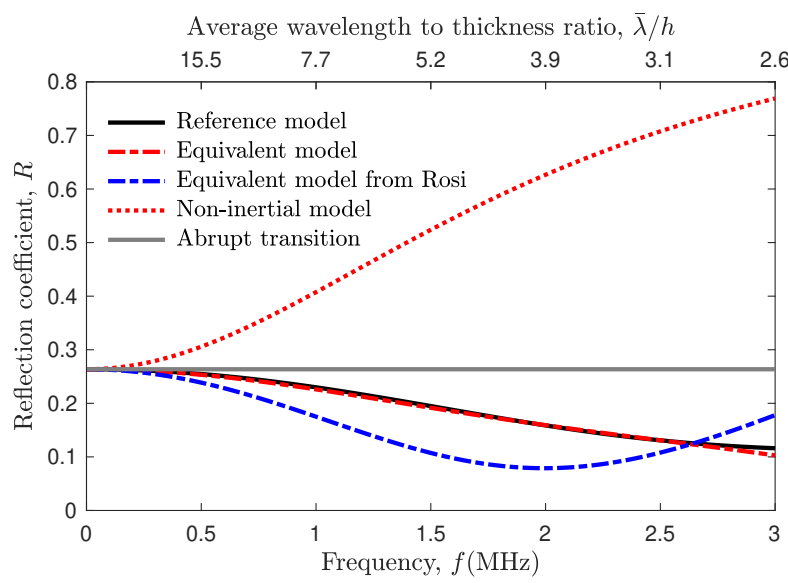

Figure 8: Frequency-dependent reflection coefficient $R$ obtained using: (1) the reference model (continuous black line); (2) an abrupt transition (continuous gray line); (3) a non-inertial equivalent interface model (dotted red line); (4) the model (dashed blue line) associated with a displacement field with an affine profile (Ref. [38]); and (5) the equivalent interface model (dashed red lines) for an optimal value of $x_{l}$ equal to 1.2 $\mu \mathrm{m}$.

optimization, our equivalent model with specific interface conditions provides a very accurate approximation of the reference model over a broad frequency range up to around $2.6 \mathrm{MHz}$. It thus outperforms the results obtained with the equivalent model from [38], which still remains valid but for a much narrower frequency regime. In contrast, an equivalent model that only accounts for elastic interface conditions totally fails in capturing the complex dynamics of the interphase. 


\section{Discussion}

Computational modeling of functionally graded intephases between soft and hard materials has provided important insights on underlying anchoring mechanisms at the microscale and on surgical repair strategies at the macroscale. To unravel additional mechanical characteristics related to the functioning of such complex interphases, as well as to enhance the characterization of bioinspired attachment procedures or tissue engineered implants, current models should include a precise description of the graded mechanical behavior of the interphase across several length scales. However, such modeling strategy raises several challenges from a computational viewpoint, especially in the case of dynamic loading.

In this study, we proposed an equivalent model based on surface elasticity, in which the finite thickness, continuous functionally graded interphase between the tendon and bone can be substituted by a zero-thickness interface. This was achieved by replacing the kinetic and potential energies of the interphase by specific surface energy density terms, whose coefficients could be identified in a straightforward manner using known profiles for the effective stiffness and density across the interphase. With respect to previously reported models, this novel formulation introduces an additional degree-of-freedom, which allows for a more refined description of the mechanical fields (e.g., displacements and velocities) across the interphase. The performance of the model was evaluated in the context of wave propagation.

The main findings from this numerical study were as follows: (1) as the performance of enriched models generally depends upon a proper selection of the kinematics across the interphase, our numerical results showed that the displacement field could be adequately modeled as a two-piece profile associated with an additional degree-of-freedom; (2) the assessment of the frequency-dependent reflection coefficient showed that our optimized enriched model can provide a very accurate approximation of the reference model over a broad frequency range up to around $2.6 \mathrm{MHz}$, thus outperforming the results obtained with more simplistic models; and (3) this model has a much lower computational cost than the reference one, as the interphase must not be incorporated (i.e., meshed) explicitly. Overall, our numerical results showed that the proposed approach is well-suited for the solution of a complete elastodynamics problem in the frequency-domain, as it captures the complex mechanical behavior of the tendon-to-bone interphase over a broad frequency range. Interestingly, all these significant outcomes are reached based on the optimal position of the additional degree-of-freedom, whose choice may depend on several parameters, among which the underlying behavior of the gradient in mechanical properties across the interphase is thought to be a determinant factor.

To test this hypothesis, we also investigated the impact of microstructural damage at the tendon-bone interphase on the optimal position of the additional degree-of-freedom (see Fig. 9). Clinical burden related to physical overloading, disuse or systemic diseases may manifest as a delayed onset of mineralization and an increasing angular deviation from the main collagen fibers direction across the tendon-to-bone insertion [46-48], which in turn lead to modified graded mechanical properties at the macroscale [14, 39]. A can be observed, for such a damaged configuration the gradient in stiffness displays a slightly shifted percolation 

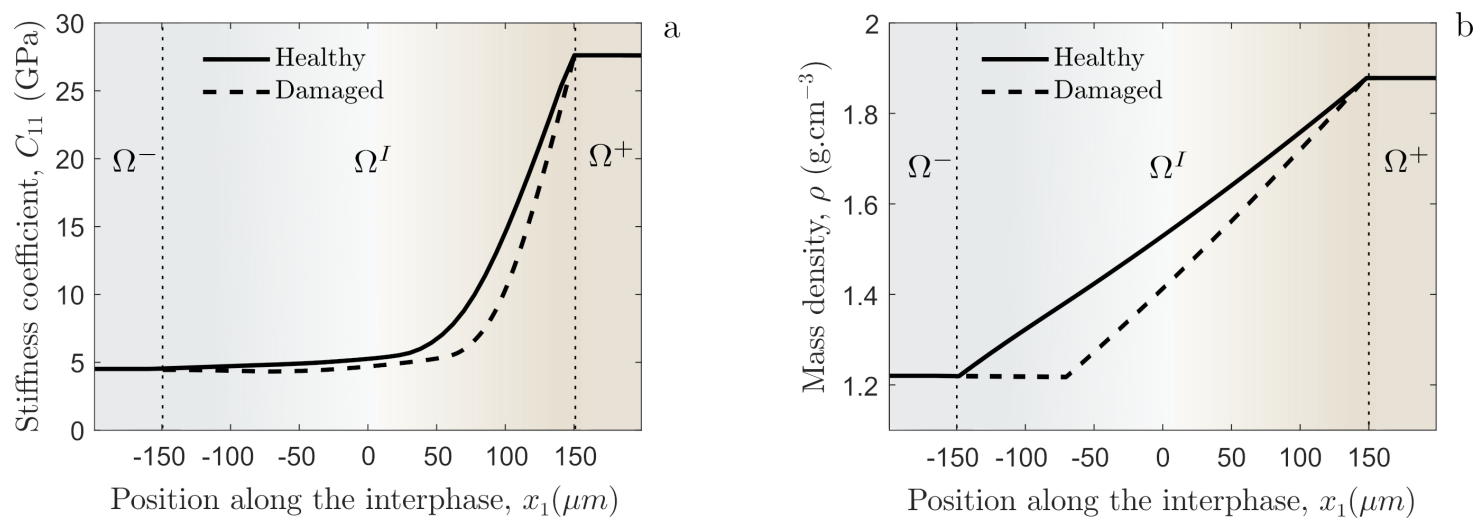

Figure 9: Graded mechanical properties across the tendon-to-bone interphase representative of a damaged configuration (dashed lines): (a) effective stiffness coefficient $C_{11}$ and (b) effective mass density $\rho$. The nominal case from Fig. 5 is shown for comparison (continuous lines).

threshold with respect to the nominal case (see Fig. 9a), whereas the gradient in mass density now has a piece-wise profile (see Fig. 9b).

Using these modified graded profiles as input of our enriched model and applying the optimization procedure described in Subsect. 3.2 yield different values for the optimal position $\hat{x}_{l}$ and for the identified coefficients associated with the surface energy density terms (see Tab. 2). Interestingly, the optimal position $\hat{x}_{l}$ has now raised to around $9 \mu \mathrm{m}$ with respect to its nominal value, and this shift is of the same order than that related to the percolation threshold observed in the stiffness profile (see Fig. 9a). Beside the shift of $\hat{x}_{l}$, the identified coefficients are also impacted, but their interpretation is not straightforward and we postpone this analysis to future works. Nevertheless, our model proves to be sensitive to slight

\begin{tabular}{|c|c|c|c|c|c|c|c|c|}
\hline \multirow[b]{2}{*}{ Case } & \multirow{2}{*}{$\begin{array}{c}\text { Position } \\
\hat{x}_{l} \\
\mu \mathrm{m}\end{array}$} & \multicolumn{3}{|c|}{ Surface mass densities } & \multicolumn{2}{|c|}{ Kinetic interactions } & \multicolumn{2}{|c|}{ Surface stiffnesses } \\
\hline & & $m^{-}$ & $\begin{array}{c}m^{+} \\
\text {kg.m }\end{array}$ & $m_{l}$ & $\gamma_{1}$ & $a^{-2}{ }^{\gamma_{2}}$ & $\begin{array}{l}K_{1} \\
\text { kg.(s }\end{array}$ & $\begin{array}{l}K_{2} \\
2 \times 10^{13}\end{array}$ \\
\hline Health & 1.2 & 0.0653 & 0.0881 & 0.1525 & 0.0346 & 0.0418 & 3.0 & 8.05 \\
\hline Damaged & 10.3 & 0.0658 & 0.0818 & 0.1427 & 0.0339 & 0.0384 & 2.72 & 7.57 \\
\hline
\end{tabular}

Table 2: Comparison between the optimal positions of the additional degree-of-freedom and the coefficients of the enriched model for the healthy and damaged cases.

changes of the graded properties across the interphase (see Fig. 10) and could potentially be used for characterization purposes. 


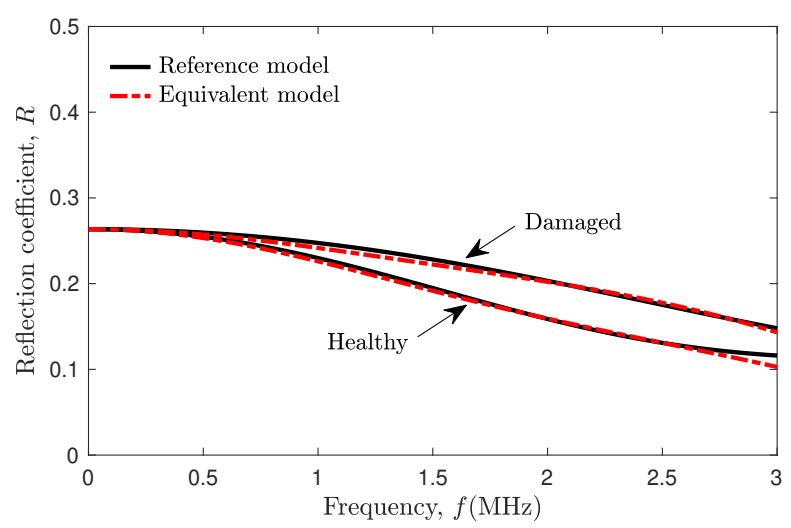

Figure 10: Comparison between the reference model (continuous black lines) and the optimal equivalent interface model (dashed red lines) for two different gradients in mechanical properties across the tendon-tobone interphase (recall Fig. 9).

Although the proposed enriched framework was established to account for a general 3-D formulation, the investigated configuration was subsequently reduced to the rather simple case of a plane elastic wave propagating under normal incidence across a functionally graded interphase, whose mechanical properties vary with respect to a single direction only. In a such case, the reflection/transmission problem is limited to the analysis of longitudinal bulk waves. Therefore, to further tackle the complexity of the tendon-to-bone attachment, whose geometry, for instance at the Achilles tendon, is characterized by a shallow attachment angle and an optimized shape of the outward splay, as well as by interdigitation mechanisms (recall Fig. 1a-b) [49, 50], future modeling efforts should consider 2-D geometries using plane wave propagating under oblique incidence (i.e., both longitudinal and shear waves) or surface waves [51]. As a further drawback, the performance of the enriched model was here only assessed based on the analysis of the frequency-dependent reflection coefficient, thus only displaying information on the amplitude spectrum of the propagating waves. However, the phase of the waves plays a crucial role as well, especially for the analysis of time-domain signals traveling across thin interphases. Further studies are warranted to precisely address the impact of the equivalent modeling approach on the phase spectrum. Overall this modeling approach represents the first critical step towards the development of more sophisticated models targeting mechanical strategies for improving diagnostic and reattachment procedures, which will not only be valuable for evaluating the tendon-to-bone insertion, but also other multiscale and graded biological attachments such as the bone-implant interphase [52, 53] and new generations of engineered composite materials [54].

\section{Conclusion}

The original equivalent interface model presented in this study aimed at mimicking the elastodynamic behavior of a finite heterogeneous interphase, while reducing the overall computational burden related to the consideration of its complete geometry. It has been shown that, in the case of a dynamic experiment, i.e., plane pressure waves under normal incidence, 
the proposed enriched model remains valid over a broad frequency range and outperforms more simplistic models that typically serve as a baseline in FE simulations at the organ scale. The key feature of the model was the introduction of an additional degree of freedom in the interface, which allowed for a finer description of the kinematic fields within the interphase that accounted for both its inertial behavior and the kinetic interactions with the surrounding media. Furthermore, it has been shown that the model was sensitive to slight variations of the graded mechanical properties across the interphase, thus opening potential applications in the field of nondestructive testing and characterization. Finally, it should be noted that such modeling approach is straightforwardly extendable to account for more complex interphase geometries.

\section{Acknowledgements}

This work was partially funded by the BEST-AMUS project (IIN program, CNRSINSIS), the "Support for research for newly appointed Associate Professors" and the "Bonus Qualité Recherche" (Faculté des Sciences et Technologie, Université Paris-Est Créteil).

\section{Appendix A. Equivalent interface model used for comparison}

Here we briefly recall the underlying equations of the equivalent interface model introduced in [38]. In that study, the displacement field was associated with an affine profile. As such, the general forms for the kinetic and potential energies per unit surface were stated as

$$
\begin{aligned}
T^{S} & =\frac{1}{2}\left[m^{+}\left(v^{+}\right)^{2}+m^{-}\left(v^{-}\right)^{2}+2 \gamma v^{+} v^{-}\right], \\
U^{S} & =\frac{1}{2} K\left(v^{+}-v^{-}\right) \cdot\left(v^{+}-v^{-}\right),
\end{aligned}
$$

where the coefficients to be identified are

$$
m^{ \pm}=\frac{M_{0}}{4} \pm \frac{M_{1}}{h}+\frac{M_{2}}{h^{2}}, \quad \gamma=\frac{M_{0}}{4}-\frac{M_{2}}{h^{2}},
$$

in which $M_{\alpha}$ represents the $\alpha$-th order moment of the density profile of the interphase

$$
M_{\alpha}=\int_{0}^{h} x_{1}^{\alpha} \rho^{I}\left(x_{1}\right) d x_{1}, \quad \text { for } \quad \alpha=0,1,2 .
$$




\section{References}

[1] S. Thomopoulos, V. Birman, and G. M. Genin. The challenge of attaching dissimilar materials. In Structural Interfaces and Attachments in Biology, pages 3-17. Springer, 2013.

[2] A. Sola, D. Bellucci, and V. Cannillo. Functionally graded materials for orthopedic applications-an update on design and manufacturing. Biotechnol. Adv., 34(5):504-531, 2016.

[3] J. W. Y. Jor, M. D. Parker, A. J. Taberner, M. P. Nash, and P. M. F. Nielsen. Computational and experimental characterization of skin mechanics: identifying current challenges and future directions. WIRES Syst. Biol. Med., 5(5):539-556, 2013.

[4] V. Bousson, A. Meunier, C. Bergot, É. Vicaut, M. A. Rocha, M. H. Morais, A.-M. Laval-Jeantet, and J.-D. Laredo. Distribution of intracortical porosity in human midfemoral cortex by age and gender. $J$. Bone Miner. Res., 16(7):1308-1317, 2001.

[5] J. Apostolakos, T. J. S. Durant, C. R. Dwyer, R. P. Russell, J. H. Weinreb, F. Alaee, K. Beitzel, M. B. McCarthy, M. P. Cote, and A. D. Mazzocca. The enthesis: a review of the tendon-to-bone insertion. Muscles Ligaments Tendons J., 4(3):333, 2014.

[6] M. J. Mirzaali, A. H. de la Nava, D. Gunashekar, M. Nouri-Goushki, R. P. E. Veeger, Q. Grossman, L. Angeloni, M. K. Ghatkesar, L. E. Fratila-Apachitei, D. Ruffoni, E. L. Doubrovski, and A. A. Zadpoor. Mechanics of bioinspired functionally graded soft-hard composites made by multi-material 3D printing. Compos. Struct., page 111867, 2020.

[7] A. Tits and D. Ruffoni. Joining soft tissues to bone: insights from modeling and simulations. Bone Rep., page 100742, 2020.

[8] L. Rossetti, L. A. Kuntz, E. Kunold, J. Schock, K. W. Müller, H. Grabmayr, J. Stolberg-Stolberg, F. Pfeiffer, S. A. Sieber, R. Burgkart, and A. R. Bausch. The microstructure and micromechanics of the tendon-bone insertion. Nat. Mater., 16(6):664, 2017.

[9] J. W. C. Dunlop, R. Weinkamer, and P. Fratzl. Artful interfaces within biological materials. Mater. Today, 14(3):70-78, 2011.

[10] A. Seidi, M. Ramalingam, I. Elloumi-Hannachi, S. Ostrovidov, and A. Khademhosseini. Gradient biomaterials for soft-to-hard interface tissue engineering. Acta Biomater., 7(4):1441-1451, 2011.

[11] Z. Liu, M. A. Meyers, Z. Zhang, and R. O. Ritchie. Functional gradients and heterogeneities in biological materials: Design principles, functions, and bioinspired applications. Prog. Mater. Sci., 88:467-498, 2017.

[12] E. Gracey, A. Burssens, I. Cambré, G. Schett, R. Lories, I. B. McInnes, H. Asahara, and D. Elewaut. Tendon and ligament mechanical loading in the pathogenesis of inflammatory arthritis. Nat. Rev. Rheumatol., pages 1-15, 2020.

[13] G. M. Genin, A. Kent, V. Birman, B. Wopenka, J. D. Pasteris, P. J. Marquez, and S. Thomopoulos. Functional grading of mineral and collagen in the attachment of tendon to bone. Biophys. J., 97(4):976985, 2009.

[14] Y. Liu, S. Thomopoulos, C. Chen, V. Birman, M. J. Buehler, and G. M. Genin. Modelling the mechanics of partially mineralized collagen fibrils, fibres and tissue. J. R. Soc. Interface, 11(92):20130835, 2014.

[15] F. Saadat, V. Birman, S. Thomopoulos, and G. M. Genin. Effective elastic properties of a composite containing multiple types of anisotropic ellipsoidal inclusions, with application to the attachment of tendon to bone. J. Mech. Phys. Solids, 82:367-377, 2015.

[16] Y. Hu, V. Birman, A. Deymier-Black, A. G. Schwartz, S. Thomopoulos, and G. M. Genin. Stochastic interdigitation as a toughening mechanism at the interface between tendon and bone. Biophys. J., 108(2):431-437, 2015.

[17] Y. X. Liu, S. Thomopoulos, V. Birman, J.-S. Li, and G. M. Genin. Bi-material attachment through a compliant interfacial system at the tendon-to-bone insertion site. Mech. Mater., 44:83-92, 2012.

[18] C. Quental, J. Folgado, J. Monteiro, and M. Sarmento. Full-thickness tears of the supraspinatus tendon: A three-dimensional finite element analysis. J. Biomech., 49(16):3962-3970, 2016.

[19] M. Mantovani, A. Pellegrini, P. Garofalo, and P. Baudi. A 3D finite element model for geometrical and mechanical comparison of different supraspinatus repair techniques. J. Shoulder Elbow Surg., 25(4):557-563, 2016. 
[20] A. Shafiei, J. W. Pro, R. Martini, and F. Barthelat. The very hard and the very soft: Modeling bio-inspired scaled skins using the discrete element method. J. Mech. Phys. Solids, 146:104176, 2021.

[21] E. I. Avgoulas, M. P. F. Sutcliffe, S. W. Linderman, V. Birman, S. Thomopoulos, and G. M. Genin. Adhesive-based tendon-to-bone repair: failure modelling and materials selection. J. R. Soc. Interface, 16(153):20180838, 2019.

[22] S. Kuznetsov, M. Pankow, K. Peters, and H.-Y. S. Huang. A structural-based computational model of tendon-bone insertion tissues. Math. Biosci., 327:108411, 2020.

[23] R. Vayron, V.-H. Nguyen, R. Bosc, S. Naili, and G. Haïat. Finite element simulation of ultrasonic wave propagation in a dental implant for biomechanical stability assessment. Biomech. Model Mechanobiol., 14(5):1021-1032, 2015.

[24] M. E. Gurtin and A. I. Murdoch. A continuum theory of elastic material surfaces. Arch. Ration. Mech. Anal., 57(4):291-323, 1975.

[25] P. Bövik. On the modelling of thin interface layers in elastic and acoustic scattering problems. Q. J. Mech. Appl. Math., 47(1):17-42, 1994.

[26] Z. Hashin. Thin interphase/imperfect interface in elasticity with application to coated fiber composites. J. Mech. Phys. Solids, 50(12):2509-2537, 2002.

[27] Y. Benveniste. A general interface model for a three-dimensional curved thin anisotropic interphase between two anisotropic media. J. Mech. Phys. Solids, 54(4):708-734, 2006.

[28] S. T. Gu and Q.-C. He. Interfacial discontinuity relations for coupled multifield phenomena and their application to the modeling of thin interphases as imperfect interfaces. J. Mech. Phys. Solids, 59(7):1413$1426,2011$.

[29] G. Mishuris. Imperfect transmission conditions for a thin weakly compressible interface. $2 \mathrm{~d}$ problems. Arch. Mech., 56(2):103-115, 2004.

[30] M. Sonato, A. Piccolroaz, W. Miszuris, and G. Mishuris. General transmission conditions for thin elasto-plastic pressure-dependent interphase between dissimilar materials. Int. J. Solids Struct., 64:921, 2015.

[31] D. Bigoni and A. B. Movchan. Statics and dynamics of structural interfaces in elasticity. Int. J. Solids Struct., 39(19):4843-4865, 2002.

[32] M. Brun, S. Guenneau, A. B. Movchan, and D. Bigoni. Dynamics of structural interfaces: filtering and focussing effects for elastic waves. J. Mech. Phys. Solids, 58(9):1212-1224, 2010.

[33] M. Touboul, B. Lombard, and C. Bellis. Time-domain simulation of wave propagation across resonant meta-interfaces. J. Comput. Phys., 414:109474, 2020.

[34] M. Touboul, K. Pham, A. Maurel, J.-J. Marigo, B. Lombard, and C. Bellis. Effective resonant model and simulations in the time-domain of wave scattering from a periodic row of highly-contrasted inclusions. J. Elast., 142(1):53-82, 2020.

[35] F. dell'Isola, A. Madeo, and L. Placidi. Linear plane wave propagation and normal transmission and reflection at discontinuity surfaces in second gradient 3D continua. ZAMM-Journal of Applied Mathematics and Mechanics/Zeitschrift für Angewandte Mathematik und Mechanik, 92(1):52-71, 2012.

[36] L. Placidi, G. Rosi, I. Giorgio, and A. Madeo. Reflection and transmission of plane waves at surfaces carrying material properties and embedded in second-gradient materials. Math. Mech. Solids, 19(5):555$578,2014$.

[37] I. Scala, G. Rosi, L. Placidi, V.-H. Nguyen, and S. Naili. Effects of the microstructure and density profiles on wave propagation across an interface with material properties. Continuum Mech. Therm., 31(4):1165-1180, 2019.

[38] G. Rosi, L. Placidi, V.-H. Nguyen, and S. Naili. Wave propagation across a finite heterogeneous interphase modeled as an interface with material properties. Mech. Res. Commun., 84:43-48, 2017.

[39] A. Aghaei, N. Bochud, G. Rosi, and S. Naili. Assessing the effective elastic properties of the tendonto-bone insertion: a multiscale modeling approach. Biomech. Model Mechanobiol., pages 1-16, 2020.

[40] F. dell'Isola and A. Romano. On the derivation of thermomechanical balance equations for continuous systems with a nonmaterial interface. Int. J. Eng. Sci., 25(11-12):1459-1468, 1987.

[41] F. dell'Isola and A. Romano. A phenomenological approach to phase transition in classical field theory. 
Int. J. Eng. Sci., 25(11-12):1469-1475, 1987.

[42] F. dell'Isola and L. Placidi. Variational principles are a powerful tool also for formulating field theories. In Variational models and methods in solid and fluid mechanics, pages 1-15. Springer, 2011.

[43] I. Wakabayashi, E. Itoi, H. Sano, Y. Shibuya, R. Sashi, H. Minagawa, and M. Kobayashi. Mechanical environment of the supraspinatus tendon: a two-dimensional finite element model analysis. J. Shoulder Elbow Surg., 12(6):612-617, 2003.

[44] R. E. Debski, J. A. Weiss, W. J. Newman, S. M. Moore, and P. J. McMahon. Stress and strain in the anterior band of the inferior glenohumeral ligament during a simulated clinical examination. $J$. Shoulder Elbow Surg., 14(1):S24-S31, 2005.

[45] E. Pena, B. Calvo, M. A. Martinez, and M. Doblare. A three-dimensional finite element analysis of the combined behavior of ligaments and menisci in the healthy human knee joint. J. Biomech., 39(9):1686-1701, 2006.

[46] S. Thomopoulos, G. R. Williams, and L. J. Soslowsky. Tendon to bone healing: differences in biomechanical, structural, and compositional properties due to a range of activity levels. J. Biomech. Eng., 125(1):106-113, 2003.

[47] M. Benjamin, H. Toumi, J. R. Ralphs, G. Bydder, T. M. Best, and S. Milz. Where tendons and ligaments meet bone: attachment sites ('entheses') in relation to exercise and/or mechanical load. $J$. Anat., 208(4):471-490, 2006.

[48] A. C. Deymier, A. G. Schwartz, Z. Cai, T. L. Daulton, J. D. Pasteris, G. M. Genin, and S. Thomopoulos. The multiscale structural and mechanical effects of mouse supraspinatus muscle unloading on the mature enthesis. Acta. Biomater., 83:302-313, 2019.

[49] Y. Liu, V. Birman, C. Chen, S. Thomopoulos, and G. M. Genin. Mechanisms of bimaterial attachment at the interface of tendon to bone. J. Eng. Mater. Technol., 133(1):011006, 2011.

[50] A. C. Abraham and T. L. H. Donahue. From meniscus to bone: a quantitative evaluation of structure and function of the human meniscal attachments. Acta Biomater., 9(5):6322-6329, 2013.

[51] V. A. Eremeyev, G. Rosi, and S. Naili. Transverse surface waves on a cylindrical surface with coating. Int. J. Eng. Sci., 147:103188, 2020.

[52] Y. Hériveaux, V.-H. Nguyen, and G. Haïat. Reflection of an ultrasonic wave on the bone-implant interface: A numerical study of the effect of the multiscale roughness. J. Acoust. Soc. Am., 144(1):488499, 2018.

[53] S. Le Cann, E. Törnquist, I. Silva Barreto, M. Fraulob, H. A. Lomami, M. Verezhak, M. GuizarSicairos, H. Isaksson, and G. Haiat. Spatio-temporal evolution of hydroxyapatite crystal thickness at the bone-implant interface. Acta Biomater., 116:391-399, 2020.

[54] F. Xu, X. Zhang, and H. Zhang. A review on functionally graded structures and materials for energy absorption. Eng. Struct., 171:309-325, 2018. 